their adherence. Thematic analysis was conducted to examine any common themes.

Main Outcome Measurements Adapted Sports Injury Rehabilitation Adherence Scale (SIRAS) with follow up focus groups.

Results This study found that men's players had a better attendance to their performance programs than women's players. However, men's players felt they had less input into their rehabilitation sessions compared to women's players, despite having a better understanding of the exercises given. Common themes that emerged from focus groups that influenced adherence were financial pressures, experience, perceptions of pain, self-determination and staff relationship - these themes were common in both men's and women's players.

Conclusions Despite differences in funding and infrastructure, both men's and women's professional football players identify broadly the same factors that influence adherence. Particularly, both groups felt a greater understanding of financial pressures and self-determination would allow for the fostering of an adherence culture and should be considered in future program design.

\section{$314 \quad$ ABSTRACT WITHDRAWN}

\section{RECTUS FEMORIS AND ILIOPSOAS CONTRACTURE AS A RISK FACTORS FOR OSGOOD-SCHLATTER DISEASE IN HIGH PERFORMANCE YOUTH ATHLETES}

Carlos Alonso Gomez Lopez, Citlali Martinez Martinez, Humberto Lopez Garcia. Code, Instituto Deporte De Jalisco, Guadalajara, Mexico

\subsection{6/bjsports-2021-IOC.288}

Background Osgood-Schlatter disease (OSD) of the knee in youth is one of the most frequent of anterior knee pain in athletes. There are several risk factors that contribute to this disease.

Objective Rectus femoris and iliopsoas contracture is a specific risk factor for the development of OSD in high performance youth athletes which come to the Centre of Applied Sciences at the sports in Jalisco.

Design The study design was cross-sectional, observational and descriptive. Participants were patients under 16 years of age who had an orthopedic consult during 2017 and 2018 and who had clinical and radiologic diagnosis of OSD. Measurement of the contracture was completed with the patient in recumbent prone position and with the quadriceps in a stretched position: the knee was flexed until the heel touched the gluteus. The epidemiological and quantitative results were calculated using excel with a significance level of $\alpha=5 \%$.

Setting Centre of Applied Sciences for Sports in Jalisco.

Patients (or Participants) 30 patients, members of the representative teams of Jalisco

Main Outcome Measurements Knee flexion and touching the gluteus with the heel.

Results 30 patients of ages 10 to 16 years old were included in the data analysis (male $=16$, female $=14 ; M_{\text {age }}=14$ ). The spread of sports played by the patients were as follows: trampoline gymnastics $=3$, weightlifting $=7$, diving $=4$, artistic gymnastics $=3$, basketball $=4$, wrestling $=2$, judo $=1$, pentathlon $=1, \quad$ canoeing $=1, \quad \operatorname{softball}=1, \quad$ squash $=1, \quad$ waterpolo $=1$, baseball $=1$. Only $30 \%$ of the patients were able to finish the maneuver: $70 \%$ were not able to touch the gluteus with their heel.

Conclusions Low elasticity of the rectus femoris and iliopsoas as a specific risk factor for the development of OSD in youth athletes was statistically significant $(p=0.05)$. General risk factors of this disease are described in the literature, the majority of which are non-modifiable. In contrast, contracture of the quadriceps and iliopsoas are modifiable risk factors for which preventative interventions could be implemented.

\section{INJURIES ACCORDING TO THE PERCENTAGE OF ADULT HEIGHT IN AN ELITE FOOTBALL ACADEMY}

${ }^{1}$ Xabier Monasterio, 'Susana María Gil, 'Iraia Bidaurrazaga-Letona, ${ }^{1,2}$ Jose Antonio Lekue, ${ }^{1,2}$ Gontzal Diaz-Beitia, 1,2Juan Maria Santisteban, 1,2Imanol Martin-Garetxana, ${ }^{2}$ Eder Bikandi, ${ }^{2}$ Julen Arce, ${ }^{2}$ Jon Larruskain. 'University of the Basque Country (UPV/EHU), Department of Physiology, Faculty of Medicine and Nursing, Leioa, Spain; ${ }^{2}$ Athletic Club Bilbao, Lezama, Spain

\subsection{6/bjsports-2021-IOC.289}

Background Injuries have a negative impact on the development of young football players. The percentage of predicted adult height is being used as an indicator of maturity status for bio-banding, yet no study has investigated its association with injury risk in football.

Objective To establish the percentage of adult height at which the most relevant football injuries occur.

Design Prospective cohort study.

Setting Elite male football players from a Spanish LaLiga club's academy.

Patients (or Participants) 63 players were followed between 1998 and 2019, since they were on average 10.7 years old (80.8\% of adult height) until they reached their final height (one full season at 100\%).

Assessment of Risk Factors The medical staff recorded injuries following the FIFA consensus and measured height every 3-6 months.

Main Outcome Measurements The percentage of adult height at which each injury occurred was calculated using the player's closest height to the injury and his final adult height. Injuries were classified in maturity bands, Pre-peak height velocity (Pre-PHV) <88\%, Peak heigh velocity (PHV) 88-95\% and Post-peak height velocity (Post-PHV) $>95 \%$.

Results Sever's disease occurred at a median $87.8 \%$ of adult height (interquartile range: $82.3-91.5 \%$, band with the highest frequency: $58.8 \%$ pre-PHV), Osgood-Schlatter disease at $87.2 \%(85.9-91.2 \%, 54.2 \%$ pre-PHV) and anterior inferior iliac spine injuries at 91.0\% (88.9-93.8\%, 69.2\% PHV). Spondylolysis occurred at $98.7 \%$ of adult height $(97.5-99.5 \%$, 92.3\% post-PHV). Similarly, muscle and joint/ligament injuries were more common Post-PHV: hamstring injuries at $98.4 \%$ (96.6-99.4\%, 77.8\% post-PHV), quadriceps injuries at $98.5 \%$ (94.8-99.5\%, 72.4\% post-PHV), knee joint/ligament injuries at 98.9\% (98.0-99.9\%, 87.5\% post-PHV), anterior cruciate ligament ruptures at $98.6 \%(98.0-100 \%, 100 \%$ post-PHV) and ankle joint/ligament injuries at $98.3 \% \quad(89.4-99.3 \%, \quad 65.7 \%$ post-PHV).

Conclusions The percentage of predicted adult height could be determined to detect high-risk periods for specific injuries and guide preventive strategies in young footballers. 